\title{
Whole-body diffusion-weighted MRI in lymphoma-comparison of global apparent diffusion coefficient histogram parameters for differentiation of diseased nodes of lymphoma patients from normal lymph nodes of healthy individuals
}

\author{
Ricardo Donners ${ }^{1,2} \wedge$, Raphael Shih Zhu Yiin ${ }^{3}$, Dow-Mu Koh ${ }^{2,4}$, Katja De Paepe ${ }^{5}$, Ian Chau ${ }^{6}$, Sue Chua ${ }^{7}$, \\ Matthew D. Blackledge ${ }^{4}$ \\ ${ }^{1}$ Department of Radiology, University Hospital Basel, Petersgraben 4, 4031 Basel, Switzerland; ${ }^{2}$ Department of Radiology, Royal Marsden Hospital, \\ Sutton SM2 5PT, UK; ${ }^{3}$ Changi General Hospital, Simei, 529889, Singapore; ${ }^{4}$ Cancer Research UK Cancer Imaging Centre, The Institute of Cancer \\ Research, Sutton SM2 5NG, UK; ${ }^{5}$ Department of Radiology, University Hospitals Leuven, Herestaat 49, Belgium; ${ }^{6}$ Gastrointestinal and Lymphoma \\ Unit, The Royal Marsden Hospital, Surrey SM2 5PT, UK; ${ }^{7}$ Department of Nuclear Medicine and PET, Royal Marsden Hospital, Sutton SM2 5PT, UK
}

Contributions: (I) Conception and design: R Donners, RSZ Yiin, DM Koh, MD Blackledge; (II) Administrative support: R Donners, RSZ Yiin, DM Koh, MD Blackledge; (III) Provision of study materials or patients: R Donners, RSZ Yiin, DM Koh, I Chau, S Chua, MD Blackledge; (IV) Collection and assembly of data: R Donners, RSZ Yiin, MD Blackledge; (V) Data analysis and interpretation: All authors; (VI) Manuscript writing: All authors; (VII) Final approval of manuscript: All authors.

Correspondence to: Ricardo Donners, MD. Department of Radiology, University Hospital Basel, Petersgraben 4, 4031 Basel, Switzerland. Email: ricardo.donners@usb.ch.

Background: Morphologic features yield low diagnostic accuracy to distinguish between diseased and normal lymph nodes. The purpose of this study was to compare diseased lymphomatous and normal lymph nodes using global apparent diffusion coefficient (gADC) histogram parameters derived from whole-body diffusion-weighted MRI (WB-DWI).

Methods: 1.5 Tesla WB-DWI of 23 lymphoma patients and 20 healthy volunteers performed between 09/2010 and 07/2015 were retrospectively reviewed. All diseased nodal groups in the lymphoma cohort and all nodes visible on b900 images in healthy volunteers were segmented from neck to groin to generate a total diffusion volume (tDV). A connected component-labelling algorithm separated spatially distinct nodes. Mean, median, skewness, kurtosis, minimum, maximum, interquartile range (IQR), standard deviation (SD), $10^{\text {th }}$ and $90^{\text {th }}$ centile of the $\mathrm{gADC}$ distribution were derived from the $\mathrm{tDV}$ of each patient/volunteer and from spatially distinct nodes. gADC and regional nodal ADC parameters were compared between malignant and normal nodes using $t$-tests and ROC curve analyses. A $\mathrm{P}$ value $\leq 0.05$ was deemed statistically significant.

Results: Mean, median, IQR, 10th and 90th centiles of gADC and regional nodal ADC values were significantly lower in diseased compared with normal lymph nodes. Skewness, kurtosis and tDV were significantly higher in lymphoma. The SD, min and max gADC showed no significant difference between the two groups $(\mathrm{P}>0.128)$. The diagnostic accuracies of gADC parameters by AUC from highest to lowest were: 10th centile, mean, median, 90th centile, skewness, kurtosis and IQR. A 10th centile gADC threshold of $0.68 \times 10^{-3} \mathrm{~mm}^{2} / \mathrm{s}$ identified diseased lymphomatous nodes with $91 \%$ sensitivity and $95 \%$ specificity.

Conclusions: WB-DWI derived gADC histogram parameters can distinguish between malignant lymph nodes of lymphoma patients and normal lymph nodes of healthy individuals.

Keywords: Whole-body diffusion-weighted MRI; lymphoma; lymph nodes; decision-making; histogram; diffusion-weighted MRI

\footnotetext{
$\wedge$ ORCID: 0000-0002-1614-0521.
} 
Submitted Jan 13, 2021. Accepted for publication Mar 26, 2021.

doi: 10.21037/qims-21-50

View this article at: http://dx.doi.org/10.21037/qims-21-50

\section{Introduction}

Malignant lymphoma is a common, systemic malignancy that predominantly affects lymph nodes, but can occur anywhere in the body (1-3). Accurate assessment of the disease distribution, sites of involvement and tumour stage is essential for management planning (4). Imaging is critical and is usually performed with whole body coverage for primary staging and disease follow-up (5).

Lymph node assessment by conventional CT or MRI relies on size measurement thresholds and morphologic features to discriminate between diseased and normal lymph nodes. This method has limited diagnostic accuracy $(6,7)$. Fluorodeoxyglucose (FDG) PET/CT shows high diagnostic accuracy for detecting nodal and extranodal disease and is currently the imaging method of choice. However, PET/CT comes with exposure to ionising radiation and radioactivity $(2,8)$. In addition, some patients, for example diabetics with poorly controlled blood sugar, will not be suitable for FDG-PET/CT, because of decreased sensitivity for disease detection (9).

Whole body diffusion-weighted MRI (WB-DWI) is a functional imaging technique, which can overcome some of the shortcomings of PET/CT (10), and can be a fast and safe alternative (11). DWI visualizes differences of water mobility in the body. Cellular tissues (e.g., lymph nodes) return high signal, while the signal of the less cellular background tissues is suppressed. This results in a high signal contrast between lymph nodes and the surrounding tissues. The water mobility can be quantified by calculating the apparent diffusion coefficient (ADC), which was shown to inversely correlate with cellularity with good measurement repeatability allowing for tissue characterization (12-14). WB-DWI derived ADC is used as a cancer biomarker for multiple tumour entities with systemic, malignant disease manifestation such as multiple myeloma, metastatic prostate and breast cancer $(14,15)$.

WB-DWI was shown to be a feasible alternative for pretherapeutic staging and response evaluation in patients with lymphoma (11,16-18). Superior diagnostic accuracy of WBDWI over PET/CT was exhibited in lymphoma subtypes with variable FDG uptake (16). Significant differences were observed in the mean ADC values between lymphoma and normal lymph nodes on a node-by-node comparison basis (19). However, the deployment of mean ADC values for clinical nodal assessment has been limited by the overlap of these values between diseased and normal lymph nodes. To date, no previous study has evaluated the DWI property of lymph nodes on a global basis, by summating all the sites of nodal disease across the body and measuring the global disease ADC (gADC) value. Additional value of analysing ADC distribution parameters such as the median, centiles, standard deviation, skewness and kurtosis were shown to improve lymph node characterisation $(20,21)$. However, data related to the gADC histogram differences between normal lymph nodes and nodal disease in lymphoma from WB-DWI is lacking. Knowledge of these WB-DWI gADC histogram parameters may help with more accurate disease diagnosis of malignant nodal groups.

The purpose of this study was to compare diseased lymphomatous and normal lymph nodes using gADC histogram parameters derived from WB-DWI.

\section{Methods}

\section{Study design and patient population}

The study was conducted in accordance with the Declaration of Helsinki (as revised in 2013). The data reported in this retrospective study was derived from two prospective studies approved by the local research and ethics committee of the Royal Marsden Hospital. Written informed consent was obtained. In the first study, 23 patients with histopathologically proven lymphoma underwent WBMRI before and 12 weeks after chemotherapy. Only the baseline imaging of this patient cohort was reported as part of this analysis. 18-FDG PET/CT was performed in all patients, with a mean time interval between both modalities of seven days. In the second study, 20 healthy volunteers underwent WB-MRI as part of a protocol development study to establish normal findings on WB-MRI. They had to match the following inclusion criteria: no history of malignancy, previous surgery, acute inflammation or infection within four weeks prior to scanning and no shortor long-term medications. Informed written consent was obtained from all patients and volunteers. 

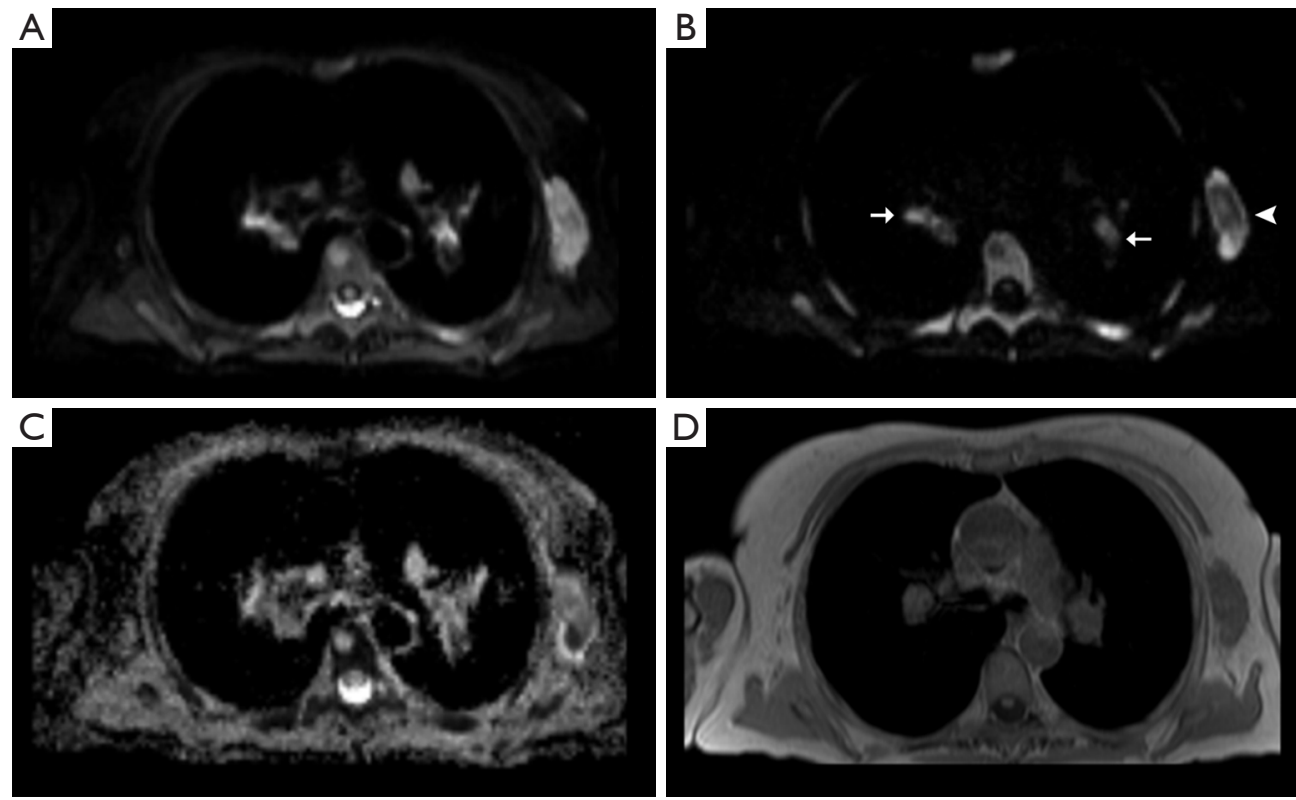

Figure 1 Whole-body diffusion-weighted MRI Protocol, axial diffusion weighted b50 (A) and b900 (B) images and the calculated apparent diffusion coefficient (ADC) map (C) show large left axillary mass (arrow head) and bilateral, hilar lymphadenopathy (arrows) with restricted diffusion in a 62-year-old male lymphoma patient. A corresponding axial T1-weighted anatomical image (D) is presented in the bottomright panel.

\section{Whole-body diffusion weighted imaging}

Axial WB-DWI of lymphoma patients and healthy individuals was performed on a $1.5 \mathrm{~T}$ MRI scanner (MAGNETOM Avanto, Siemens AG, Healthcare Sector, Erlangen, Germany) in free-breathing using the following imaging parameters: Matrix $=150 \times 144$, Partial Fourier $=6 / 8, T E=64.8 \mathrm{~ms}$, TR $=14.6 \mathrm{~s}$, Receiver Bandwidth $=1,961 \mathrm{~Hz} / \mathrm{px}$, b-values $=50$ and $900 \mathrm{~s} / \mathrm{mm}^{2}$, three scan trace weighted diffusion encoding, STIR fat suppression $(T I=180 \mathrm{~ms})$, Slice Thickness $=5 \mathrm{~mm}$, Field of View $=400 \times 390 \mathrm{~mm}^{2}$. Parallel imaging GRAPPA was applied to reduce distortion along the phase-encoding direction $(\mathrm{R}=2)$. The number of signal averages was four for each $b$-value. Images were acquired from vertex to midthigh using four consecutive imaging stations comprising of 50 slices each. The DWI acquisition time was approximately 24 minutes.

To facilitate anatomical localization of lymph nodes, breath-hold axial T1-weighted MR images, matched to the same imaging field of view and slice thickness of the WB-DWI were also acquired using the following imaging parameters: T1 fast low angle shot, matrix $=256 \times 105$, slice thickness $=5 \mathrm{~mm}, \mathrm{TR}=386 \mathrm{~ms}$, TE $=4.8 \mathrm{~ms}$, flip angle $=70^{\circ}$, number of averages $=1$. Figure 1 shows typical images obtained for a patient with lymphoma using the MRI study protocol.

\section{Image analyses and processing}

Open-access software (OsiriX version 56, PixmeoSARL Bernex, Switzerland) was used for analysis and postprocessing (22). Through visual comparison with the anatomical T1-weighted images, lymph nodes were identified on b900 images as discrete ovoid structures demonstrating high signal intensity.

\section{Global ADC (gADC) from the total disease volume}

In histopathologically proven lymphoma patients, diseased nodal groups were defined as containing nodes larger than $1 \mathrm{~cm}$ short axis diameter with visually increased $18 \mathrm{~F}-\mathrm{FDG}$ tracer uptake. All lymph nodes of malignant nodal groups visible on b900 images were included for subsequent analyses of the global ADC (gADC) histogram. Volumes of interest (VOIs) of involved lymph nodes were calculated using a proprietary IDL-based post-processing software (Exelis Visual Information Solutions, Inc.). The details for this algorithm were described by Blackledge et al. (23). 
In the volunteers, all lymph nodes visible on the b900 images were included for gADC histogram analyses. The propriety segmentation software used in lymphoma patients was not used in the volunteers, as it had not been verified for use in healthy patients. VOIs were manually generated by a board-certified radiologist using a semi-automated segmentation software employing the "GrowCUT" algorithm (24) integrated in OsiriX. VOIs were manually adjusted to include as much of the nodal parenchyma as possible, excluding non-lymphatic tissue.

The generated VOIs were summarized as the total diffusion volume (tDV) representing the total lymph node volume of each patient/volunteer and were transferred onto the corresponding ADC maps. The gADC histogram parameters mean, median, interquartile range (IQR), standard deviation (SD), 10th and 90th percentile, minimum (min) and maximum (max), skewness and kurtosis were calculated and recorded for each patient. The naturallogarithm of $\mathrm{tDV}, \ln (\mathrm{tDV})$, was calculated as an additional parameter (logarithm used to normalize the distribution of volumes).

\section{ADC from individual nodal regions}

A connected component-labelling algorithm was employed to separate spatially distinct nodal regions from the tDV in lymphoma patients and healthy volunteers. The same ADC histogram parameters described above as well as the natural logarithm of $\mathrm{tDV}, \ln (\mathrm{tDV})$, were derived from each of the spatially distinct regional nodal volumes identified using this algorithm.

\section{Statistical analyses}

Statistical analyses were performed using commercially available software (IBM SPSS Statistics Version 22, IBM Corp. Armonk, New York, USA and MedCalc Version 19.0.7, MedCalc Software bvba, Ostend, Belgium) and freeware (and RStudio, Version 1.2.1335, RStudio, Inc, Boston, USA). Unpaired $t$-tests (assuming unequal variance) were performed to compare $\ln (\mathrm{tDV})$ and gADC parameters between lymph nodes of lymphoma patients and healthy volunteers. A P value $<0.05$ was deemed statistically significant. When a parameter demonstrated statistical significance, receiver operator curves (ROCs) were generated to determine optimal parameter threshold values discriminating malignant from benign lymph nodes. Calculation of the Youden index for each point on the ROC plot allowed optimal choice of threshold values. The
ROC area under the curve (AUC) as an index of diagnostic accuracy of the test was calculated. ROC statistics were compared for ADC distribution parameters derived within the entire $\mathrm{tDV}$ and for the spatially distinct nodal volumes and a Benjamini-Hochberg procedure was used to correct for multiple comparisons. Multivariate analyses with Bonferroni corrections were performed to explore ADC differences between lymphoma subtypes.

\section{Literature review}

The PubMed database was searched for studies describing quantitative imaging analyses of lymph nodes applying $\mathrm{ADC}$ values.

\section{Results}

\section{Participants}

In total, 43 consecutive WB-DWI studies performed between 09/2010 and 07/2015 were included: pre-treatment WB-DWI of 23 patients with histopathologically verified lymphoma (12 females, 11 males) with a mean age of 59.5 years (range 24-87 years) and WB-DWI of 20 healthy individuals ( 7 female, 13 male) with a mean age of 34.8 years (range 22-60 years). Lymphoma subtypes in order of declining frequency were: diffuse large b-cell [10], follicular [7], mantle cell [3], angioimmunoblastic $T$ cell [2] and marginal cell lymphoma [1]. A total number of 1309 distinct regional nodal volumes identified on b900 images was analysed.

\section{Test results—global $A D C$}

The mean values and $\mathrm{SD}$ of $\mathrm{tDV}$ and gADC parameters calculated for each patient are shown in Table 1. We found that the mean, median, $10^{\text {th }}$ and $90^{\text {th }}$ centiles gADC values were significantly lower in malignant than in normal lymph nodes. By contrast, $\ln (\mathrm{tDV})$, gADC skewness and kurtosis were significantly larger in lymphoma. The gADC SD, IQR, minimum and maximum gADC showed no significant difference between malignant and normal lymph nodes (each $\mathrm{P}>0.052$ ).

Table 2 shows the performance of the significant gADC discriminators. The order of the diagnostic accuracies of gADC parameters by AUC performance from highest to lowest was: $10^{\text {th }}$ centile, mean, median, $90^{\text {th }}$ centile, skewness and kurtosis. Figure 2 visualises the overlap 
Table 1 Total diffusion volume and global ADC histogram comparison between lymphoma patients and healty individuals

\begin{tabular}{|c|c|c|c|c|c|}
\hline & \multicolumn{2}{|c|}{ Lymphoma } & \multicolumn{2}{|c|}{ Healthy individuals } & $P$ value \\
\hline $\ln (\mathrm{tDV})(\mathrm{mL})$ & $4.98^{*}$ & 1.25 & $3.18^{*}$ & 0.38 & 0.004 \\
\hline Mean $\left(\times 10^{-3} \mathrm{~mm}^{2} / \mathrm{s}\right)$ & $0.83^{*}$ & 0.17 & $1.15^{\star}$ & 0.13 & $<0.001$ \\
\hline $\mathrm{SD}\left(\times 10^{-3} \mathrm{~mm}^{2} / \mathrm{s}\right)$ & 0.28 & 0.09 & 0.31 & 0.05 & 0.170 \\
\hline Kurtosis & $3.76^{\star}$ & 4.13 & $1.31^{*}$ & 1.33 & 0.012 \\
\hline Median $\left(\times 10^{-3} \mathrm{~mm}^{2} / \mathrm{s}\right)$ & $0.78^{*}$ & 0.19 & $1.10^{*}$ & 0.12 & $<0.001$ \\
\hline $\operatorname{IQR}\left(\times 10^{-3} \mathrm{~mm}^{2} / \mathrm{s}\right)$ & 0.32 & 0.13 & 0.38 & 0.08 & 0.052 \\
\hline 10th centile $\left(\times 10^{-3} \mathrm{~mm}^{2} / \mathrm{s}\right)$ & $0.55^{\star}$ & 0.10 & $0.80^{*}$ & 0.09 & $<0.001$ \\
\hline Maximum $\left(\times 10^{-3} \mathrm{~mm}^{2} / \mathrm{s}\right)$ & 2.44 & 0.60 & 2.54 & 0.33 & 0.492 \\
\hline
\end{tabular}

$\mathrm{SD}$, standard deviation; IQR, interquartile range. For any parameters in which a significant difference is found between lymphoma and healthy nodes $(\mathrm{P}<0.05)$, the mean is indicated with an asterisk $\left(^{*}\right)$.

Table 2 Diagnostic performance of global ADC histogram parameters in distinguishing lymphoma from healthy lymph nodes

\begin{tabular}{lccccc}
\hline & AUC & $95 \% \mathrm{Cl}$ & Threshold & Sensitivity & Specificity \\
\hline Mean $\times 10^{-3} \mathrm{~mm}^{2} / \mathrm{s}$ & 0.92 & $0.82-0.99$ & 0.95 & 0.81 & 0.95 \\
Median $\times 10^{-3} \mathrm{~mm}^{2} / \mathrm{s}$ & 0.91 & $0.79-1.00$ & 0.91 & 0.87 & 0.91 \\
$10^{\text {th }}$ centile $\times 10^{-3} \mathrm{~mm}^{2} / \mathrm{s}$ & 0.95 & $0.86-1.00$ & 0.67 & 1.42 & 0.87 \\
$90^{\text {th }}$ centile $\times 10^{-3} \mathrm{~mm}^{2} / \mathrm{s}$ & 0.88 & $0.76-0.97$ & 1.03 & 0.65 & 0.80 \\
Skewness & 0.79 & $0.63-0.92$ & 2.04 & 0.57 & 0.95 \\
Kurtosis & 0.71 & $0.55-0.86$ & 0.95 & \\
\hline
\end{tabular}

Diagnostic descriptors are generated for apparent diffusion coeffient (ADC) parameters calculated over the entire total diffusion volume, Threshold parameter values with corresponding sensitivity and specificity are given. SD, standard deviation; IQR, interquartile range; AUC, area unter the receiver operating characteristics curve; $\mathrm{Cl}$, confidence interval.

of gADC parameters between lymphoma and healthy individuals. There was no significant difference of AUC between mean and median gADC, $10^{\text {th }}$ and $90^{\text {th }}$ centile gADC (each $\mathrm{P}>0.131$ ). Mean gADC AUC was significantly larger than gADC skewness AUC $(\mathrm{P}=0.032)$ and gADC kurtosis $(\mathrm{P}=0.004) .10^{\text {th }}$ centile and median gADC AUC were significantly larger than for gADC kurtosis (each $\mathrm{P}<0.010)$. Median and $90^{\text {th }}$ centile gADC AUC were significantly larger than for gADC skewness (each $\mathrm{P}<0.043$ ). In correspondence to the AUC, the highest Youden index among gADC histogram parameters was calculated for the $10^{\text {th }}$ centile gADC at a threshold of $0.67 \times 10^{-3} \mathrm{~mm}^{2} / \mathrm{s}$, smaller values identifying disease with $91 \%$ sensitivity and $95 \%$ specificity. A median gADC value less than $0.91 \times 10^{-3} \mathrm{~mm}^{2} / \mathrm{s}$ identified disease with $87 \%$ sensitivity and $95 \%$ specificity. $81 \%$ sensitivity and $95 \%$ specificity was shown for a threshold mean ADC value of $0.95 \times 10^{-3} \mathrm{~mm}^{2} / \mathrm{s}$. Sensitivity of the $10^{\text {th }}$ centile cut-off was significantly larger than for the mean gADC cut-off $(\mathrm{P}=0.046)$. Exemplar maximum intensity projections delineating lymph nodes of a lymphoma patient and a healthy volunteer are demonstrated in Figure 3. Figure 4 visualises the corresponding gADC histograms of lymphoma and healthy nodes of this particular patient (A) as well as the $\mathrm{ADC}$ distribution across 


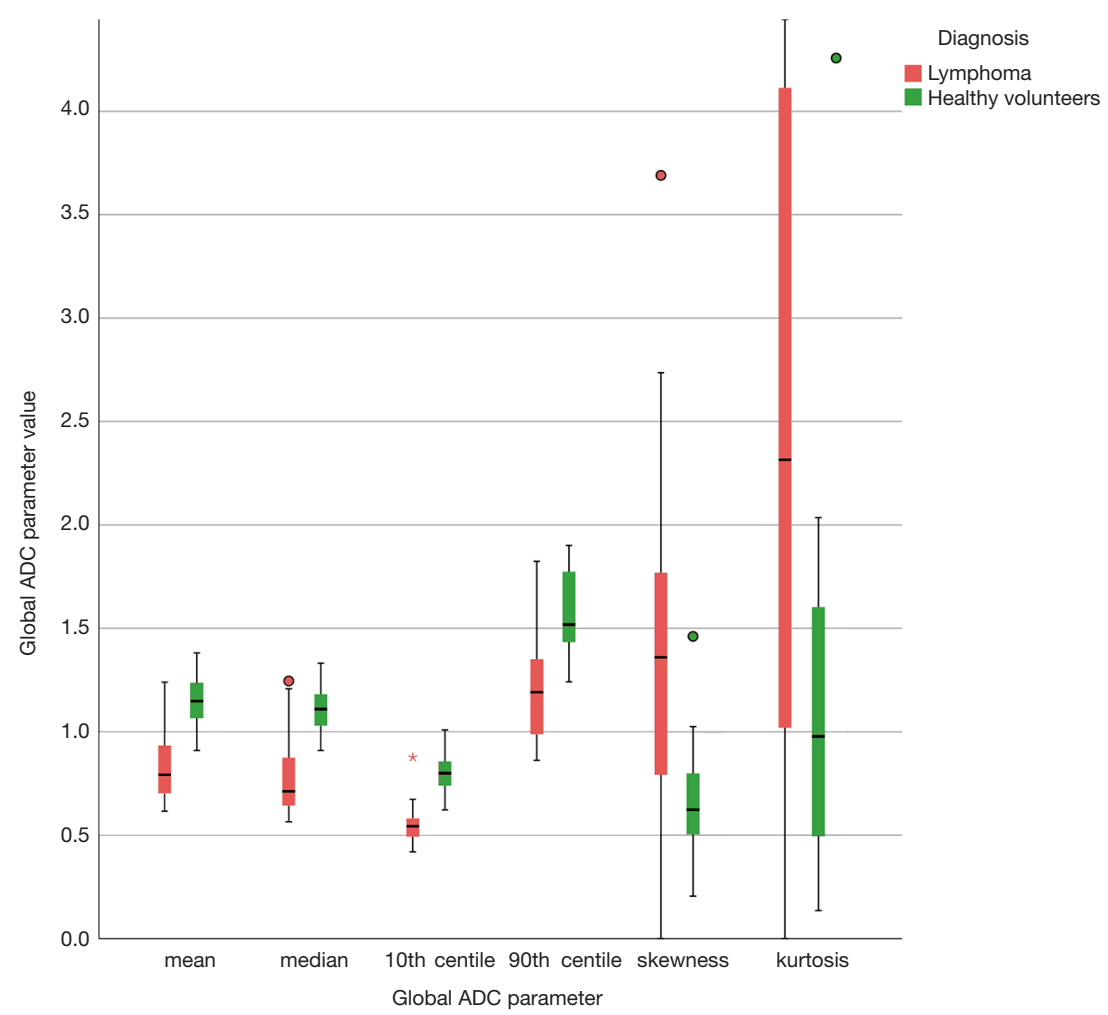

Figure 2 Global apparent diffusion coefficient (ADC) boxplots to distinguish between lymphoma and lymph node volumes of healthy volunteers. ADC values are given as $10^{-3} \mathrm{~mm}^{2} / \mathrm{s}$.

all study subjects in both groups (B). No gADC parameter showed significant difference between different lymphoma subtypes (each $\mathrm{P}>0.324$ ).

\section{Regional nodal volumes}

In total 307 diseased nodal volumes were identified in the lymphoma patients and 1,002 healthy individual nodes were identified in the volunteer group by the connected component labelling algorithm. Table 3 shows the diagnostic performance of the ADC parameters derived from distinct nodal volumes, which differed significantly between the two groups. In contrast to the corresponding gADC parameters, distinct nodal min and max ADC were significantly lower in lymphoma. The order of diagnostic accuracies of the parameters as indicated by the AUC from highest to lowest was: median and $10^{\text {th }}$ centile, mean, min, $90^{\text {th }}$ centile, skewness, kurtosis and max ADC. Violin plots demonstrating the distribution of parameters calculated for individual diseased/healthy distinct nodal volumes are compared in Figure 5. The natural logarithm of nodal volume, $\ln (\mathrm{tDV})$, showed the highest Youden index-a threshold of 0.08 (representing a $1.08 \mathrm{~mL}$ nodal volume), resulting in $75 \%$ sensitivity and $91 \%$ specificity.

\section{Literature review}

The ADC values and respective threshold values for differentiation derived from single-slice ROI measurements on small field of view head and neck MRI and other WBDWI studies are presented in Table 4.

\section{Discussion}

WB-DWI was deemed a highly sensitive imaging technique for lymphoma showing high levels of agreement with PET/ CT $(17,18,30,31,34-37)$. However, quantitative ADC thresholds for lymph node characterization are rarely applied (17). Thus, the potential of WB-DWI has not been fully exploited, in part due to the lack of established and reliable ADC threshold values discriminating between diseased and normal lymph nodes.

Our study applies an algorithm to identify and segment 

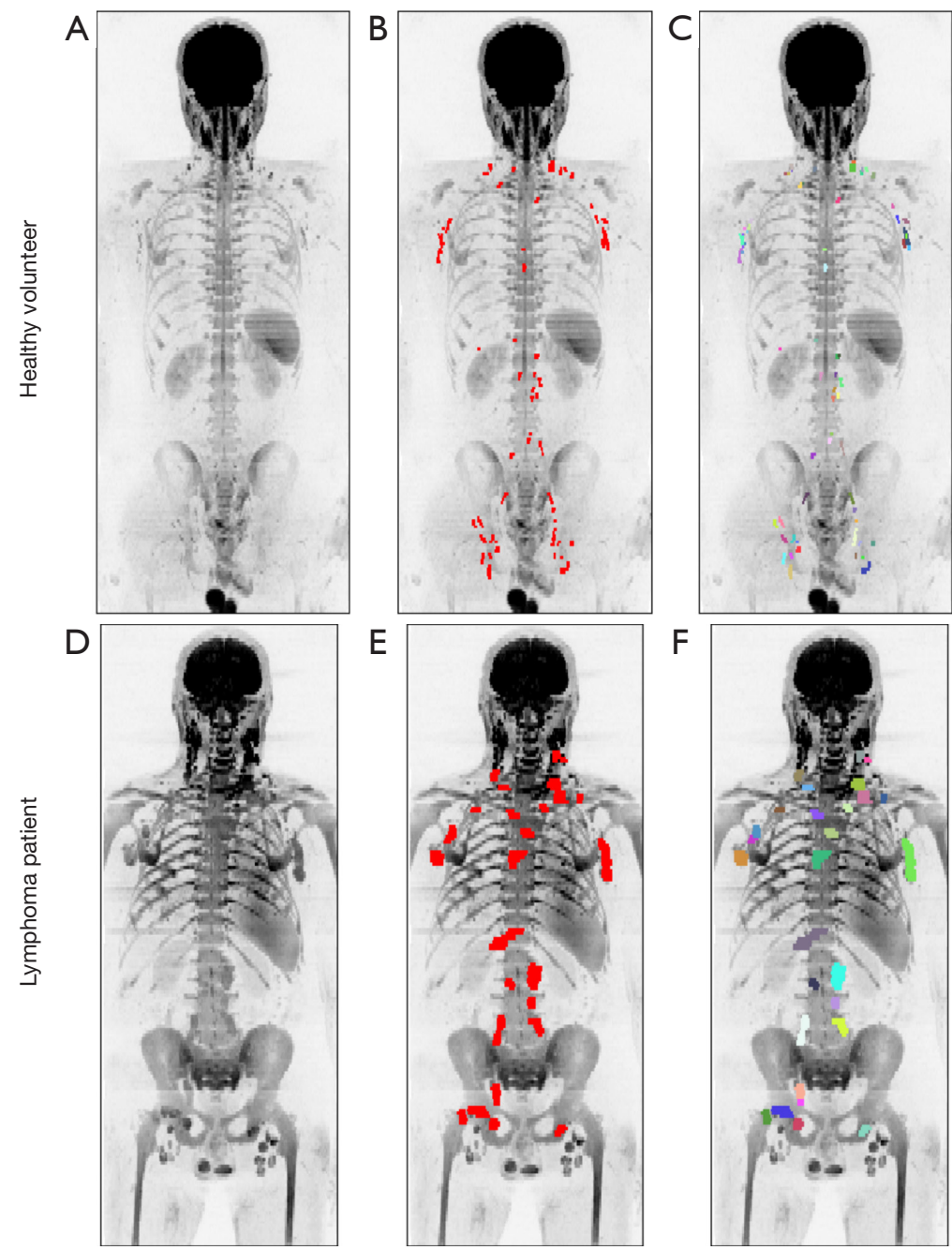

Figure 3 Volumes of interest delineating lymph nodes in a healthy volunteer and a lymphoma patient, Top: 30-year-old healthy volunteer. Maximum intensity projection (MIP) derived from the inverted b900 images (A), the superimposed red regions (B) indicate the total diffusion volume (tDV) generated by the "GrowCut" algorithm; these regions are separated using a connected component labelling algorithm and superimposed in separate colours (C). Bottom: Male 62-year-old lymphoma patient. An in-house developed algorithm was used to semi-automatically define the tDV in lymphoma patients (red regions, E). Individual nodes were segmented using the connected component labelling (multi-colour regions, F).

diseased nodal regions across the body, which allows the gADC of all lymphomatous nodes to be characterised compared with a large population of benign lymph nodes in normal volunteers. We found that gADC histogram parameters were able to distinguish between diseased lymphomatous nodes and those of healthy volunteers. The former showed significantly lower gADC values. The gADC $10^{\text {th }}$ centile performed best for discriminating between lymphoma patients and healthy volunteers. For discriminating individual nodal volumes as diseased or healthy, we found the $10^{\text {th }}$ centile value to be of good discriminatory value. Although, diagnostic performance was not significantly superior to mean ADC, this could have diagnostic implications. Previous works on different entities have described the merits of the $10^{\text {th }}$ centile ADC as an oncologic imaging biomarker, which warrants prospective performance comparison of $10^{\text {th }}$ centile, mean and median ADC (38).

In contrast to the reviewed small field of view and WB-DWI literature on quantitative DWI for lymph 
A

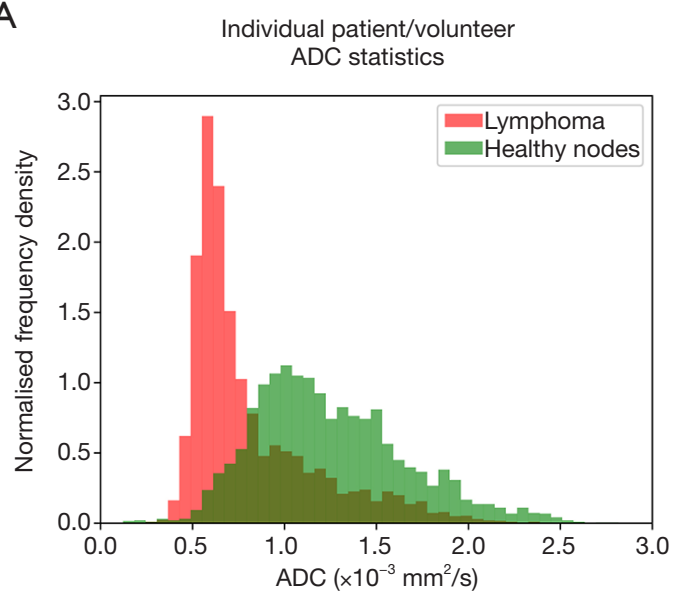

B

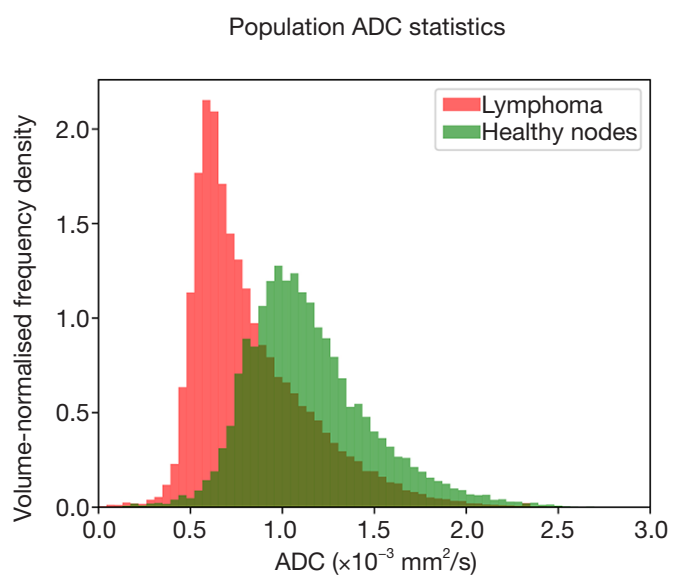

Figure 4 Global apparent diffusion coefficient (ADC) histograms. A: The healthy volunteer and the lymphoma patient whose total diffusion volumes $(\mathrm{tDV})$ are presented in Figure 3. Note the visual increase in skewness and kurtosis of the gADC distribution in lymphoma. B: Population histograms of gADC values within the $\mathrm{tDV}$ of all lymphoma patients (red) and healthy volunteers (green). To remove volume bias, histograms are composed of the weighted sum of gADC histograms for each individual patient/volunteer (weighted by the inverse of the tDV for each individual). Note the visual increase in skewness and kurtosis of the gADC distribution in lymphoma.

Table 3 Diagnostic performance of distinct nodal volume ADC histogram parameters in distinguishing lymphoma from healthy lymph nodes

\begin{tabular}{llcccc}
\hline & AUC & $95 \% \mathrm{Cl}$ & Threshold & Sensitivity & Specificity \\
\hline Mean $\times 10^{-3} \mathrm{~mm}^{2} / \mathrm{s}$ & 0.85 & $0.83-0.88$ & 0.92 & 0.71 & 0.85 \\
Median $\times 10^{-3} \mathrm{~mm}^{2} / \mathrm{s}$ & 0.86 & $0.84-0.89$ & 0.90 & 0.73 & 0.85 \\
$10^{\text {th }}$ centile $\times 10^{-3} \mathrm{~mm}^{2} / \mathrm{s}$ & 0.86 & $0.83-0.88$ & 0.70 & 0.73 & 0.86 \\
$90^{\text {th }}$ centile $\times 10^{-3} \mathrm{~mm}^{2} / \mathrm{s}$ & 0.81 & $0.78-0.84$ & 0.14 & 0.86 & 0.41 \\
Skewness & 0.64 & $0.60-0.67$ & 0.52 & 0.87 \\
Kurtosis & 0.64 & $0.61-0.68$ & 0.80 & 0.66 \\
\hline
\end{tabular}

Diagnostic descriptors are generated for apparent diffusion coeffient (ADC) parameters calculated from spatially distinct nodal volumes generated by the connected component labelling algortithm. Threshold parameter values with corresponding sensitivity and specificity are given. SD, standard deviation; IQR, interquartile range; AUC, area unter the receiver operating characteristics curve; CI, confidence interval.

node characterisation, we evaluated ADC measurements derived from a semi-automatic segmentation of lymph node volumes. In metastatic bone disease this approach was shown to offer good reproducibility (39). The calculated gADC and ADC values of normal and diseased nodes are comparable with $\mathrm{ADC}$ measurements recorded in previous studies.

Kwee et al. published the only prior study comparing malignant nodes with normal nodes of healthy patients employing WB-DWI in lymphoma patients and extended field of view DWI in healthy individuals. ADC measurements were derived from manually placed single slice ROIs.
ROIs were placed in FDG-PET/CT positive lymph nodes in lymphoma patients and in the largest lymph node of healthy patients (19). DWI of the thorax and abdomen were not included in healthy patients compared to a true WBDWI protocol employed in our volunteers. This may have contributed to a higher mean nodal gADC in our healthy volunteers, as well as a higher gADC threshold in our study compared with those reported by Kwee et al. Lymph nodes of the thorax and abdomen are prone to be affected by bulk motion, which can increase ADC values due to partial volume effects.It needs to be noted that our measured gADC values were within the standard deviation of the ADC measurements 

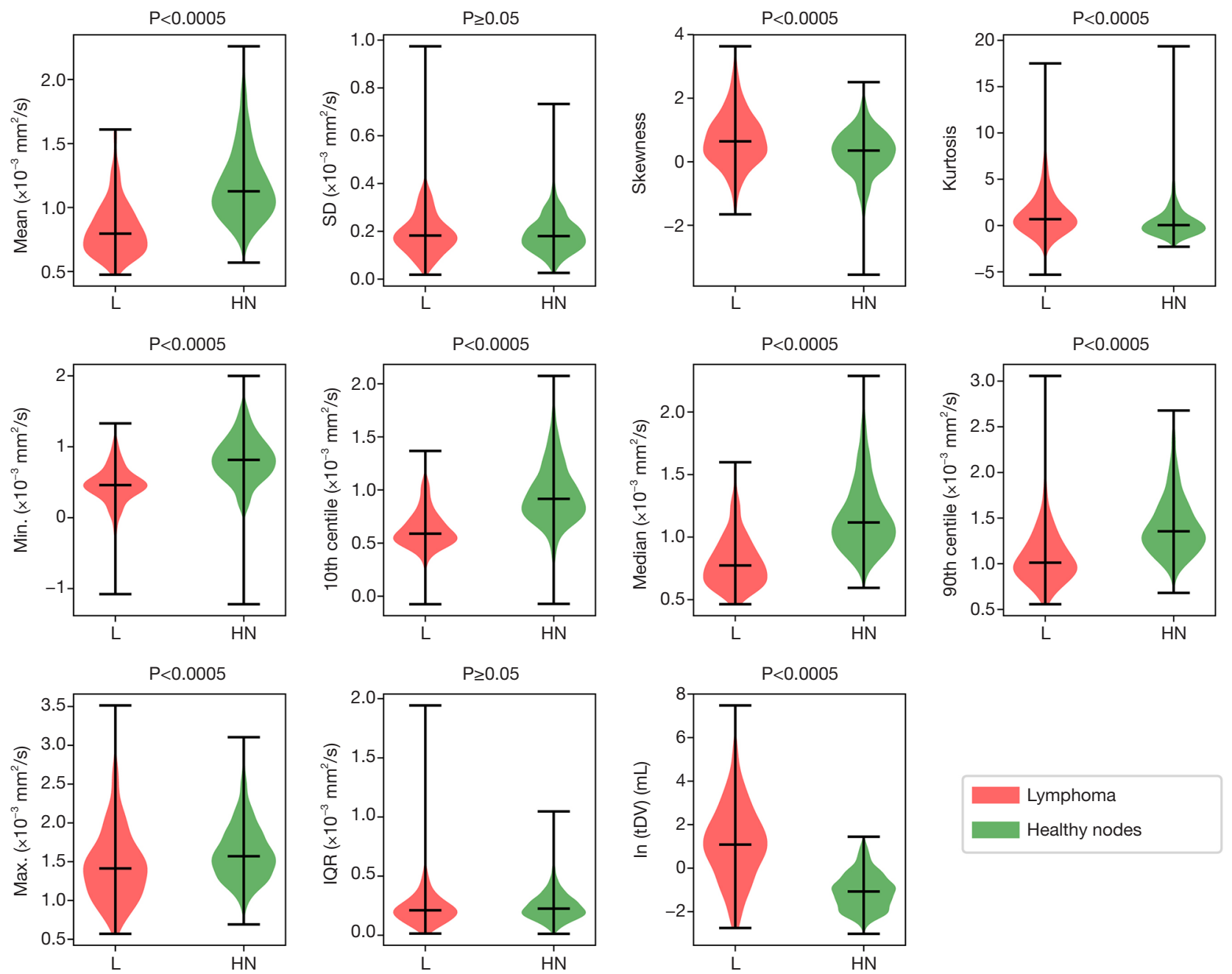

Figure 5 Violin plots for apparent diffusion coefficient (ADC) distributions derived within nodes from lymphoma patients and healthyvolunteers. In each case the shaded region demonstrates (vertically) the distribution of each statistic amongst the individual nodes (307 diseased nodes and 1002 healthy nodes). The whiskers represent (in order from bottom to top) the minimum, the median, and the maximum of the statistic.

reported by Kwee et al. Moreover, ADC values are known to have a variability of $5-12 \%$, increasing with smaller volume measurements $(40,41)$. The general agreement of gADC measurements of lymph nodes with previous literature using regional ADC measurements shows that a volume based approach is feasible and reliable while allowing to include a larger disease volume. In a future prospective scenario, the software could allow for whole-body lymph node region delineation and automated identification of suspicious nodal regions according to their $\mathrm{ADC}$ histogram characteristics and verify the derived ADC cut-off values of this study.

This study has several limitations. First, it needs to be emphasized, that within our study individual nodal volumes in lymphoma patients do not represent individual nodes, but rather the smallest individual nodal volume, as identified by the segmentation algorithm. Yet to be developed, advanced, automated segmentation tools may allow for improved identification of individual nodes and true per nodal analyses. Second, only 23 lymphoma patients were included. Consequently, ADC differences between lymphoma subtypes could not be adequately assessed. However, current literature suggests little difference in the ADC values between malignant lymph nodes of different lymphoma subtypes, which is in keeping with the presented findings 
Table 4 Literature on quantitative ADC analyses of malignant lymph nodes

\begin{tabular}{|c|c|c|c|c|c|}
\hline Authors & Mean ADC lymphoma & Mean ADC normal & Threshold ADC & Sens. in \% & Spec. in \% \\
\hline King et al. (25) & $0.66 \pm 0.07$ & & & & \\
\hline Abdel et al. (26) & $0.97 \pm 0.27$ & $1.64 \pm 0.16^{\dagger}$ & 1.38 & 98 & 88 \\
\hline Holzapfel et al. (27) & $0.64 \pm 0.09$ & $1.24 \pm 0.16^{\dagger}$ & 1.02 & 100 & 87 \\
\hline \multicolumn{6}{|l|}{ Whole body DWI } \\
\hline Chen et al. (29) & $0.60 \pm 0.11$ & & & & \\
\hline Lin et al. (30) & $0.66 \pm 0.15$ & & & & \\
\hline Wu et al. (31) & $0.71^{*}$ & & & & \\
\hline Li et al. (33) & $0.87 \pm 0.17$ & $1.20 \pm 0.10^{\dagger}$ & 1.08 & 88 & 91 \\
\hline De Paepe (21) & $1.04(0.63-3.22)$ & $1.37(0.7-2.67)^{\dagger}$ & 1.11 & 58 & 74 \\
\hline
\end{tabular}

The mean apparent diffusion coefficient (ADC) of malignant nodes in lymphoma and normal nodes; and theshold values for differentiation with corresponding sensitivity (sens) and specificity (spec) are given. ${ }^{\dagger}$ indicates that normal measurements are derived from lymph nodes deemed normal on the basis of other imaging modalities in lymphoma patients. *no standard deviation or range provided.

$(16,42)$. Third, differences in diffusion characteristics of different anatomical regions were not assessed. Fourth, we were unable to obtain pathological verification of all nodes that were segmented by DWI. However, the nodal groups segmented were verified to be FDG avid. Fifth, DWI may be subject to a number of artefacts, which affect image quality and ADC calculation. Moreover attention should be paid on the choice of b-values for ADC calculation, as low b-value $\left(<150 \mathrm{~s} / \mathrm{mm}^{2}\right)$ imaging is subject to perfusion effects, which may bias subsequent ADC calculation towards higher values. This can also have implications for ADC comparison. However, the choice of the paired b-values of $\mathrm{b}=50$ and $900 \mathrm{~s} / \mathrm{mm}^{2}$ are widely employed in whole body MRI studies. Lastly, two different segmentation methods were applied, as there was no automated segmentation tool available for normal lymph nodes at the time of the study. However, segmentation was performed by an experienced radiologist with more than 5-year experience in MRI.

In conclusion, WB-DWI derived gADC histogram parameters can distinguish between malignant lymph nodes of lymphoma patients and normal lymph nodes of healthy individuals. The $10^{\text {th }}$ centile gADC showed superior sensitivity over the mean gADC. Parameters describing the gADC histogram may serve as valuable biomarkers for lymphoma disease assessment.

\section{Acknowledgments}

Funding: This work was supported by Cancer Research UK and The Engineering and Physical Sciences Research Council's support to the Cancer Imaging Centre at ICR and $\mathrm{RMH}$ in association with the Medical Research Council and the Department of Health C1060/A10334, C1060/A16464 Invention for Innovation, Advanced computer diagnostics for whole body magnetic resonance imaging to improve management of patients with metastatic bone cancer II-LA0216-20007, and NHS funding to the NIHR Biomedical Research Centre, Clinical Research Facility in Imaging and the Cancer Research Network. Support was also received from NIHR Postdoctoral Fellowship NHR011X and this research was partly supported by the "Foundation of the Swiss Society of Radiology for Research, Postgraduate and Continuing Medical Education" and "Research Fund for excellent Junior Researchers of the University of Basel". Sponsoring bodies had no impact on study design; in the collection, analysis and interpretation of data; in the writing of the report; or in the decision to submit the article for publication. The views expressed in this manuscript are 
exclusively those of the authors.

\section{Footnote}

Conflicts of Interest: All authors have completed the ICMJE uniform disclosure form (available at http://dx.doi. org/10.21037/qims-21-50). Dr. Matthew Blackledge reports that he received patents broadly related to the field of whole-body imaging. The other authors have no conflicts of interest to declare.

Ethical Statement: The authors are accountable for all aspects of the work in ensuring that questions related to the accuracy or integrity of any part of the work are appropriately investigated and resolved. The study was conducted in accordance with the Declaration of Helsinki (as revised in 2013). The data reported in this retrospective study was derived from two prospective studies approved by the local research and ethics committee of the Royal Marsden Hospital. Written informed consent was obtained.

Open Access Statement: This is an Open Access article distributed in accordance with the Creative Commons Attribution-NonCommercial-NoDerivs 4.0 International License (CC BY-NC-ND 4.0), which permits the noncommercial replication and distribution of the article with the strict proviso that no changes or edits are made and the original work is properly cited (including links to both the formal publication through the relevant DOI and the license). See: https://creativecommons.org/licenses/by-nc-nd/4.0/.

\section{References}

1. Huh J. Epidemiologic overview of malignant lymphoma. Korean J Hematol 2012;47:92-104.

2. Gu J, Chan T, Zhang J, Leung AY, Kwong YL, Khong PL. Whole-body diffusion-weighted imaging: the added value to whole-body MRI at initial diagnosis of lymphoma. AJR Am J Roentgenol 2011;197:W384-91.

3. Evans LS, Hancock BW. Non-Hodgkin lymphoma. Lancet 2003;362:139-46.

4. Carbone PP, Kaplan HS, Musshoff K, Smithers DW, Tubiana M. Report of the Committee on Hodgkin's Disease Staging Classification. Cancer Res 1971;31:1860-1.

5. Cheson BD. Staging and response assessment in lymphomas: the new Lugano classification. Chin Clin Oncol 2015;4:5.
6. Herneth AM, Mayerhoefer M, Schernthaner R, Ba-Ssalamah A, Czerny C, Fruehwald-Pallamar J. Diffusion weighted imaging: lymph nodes. Eur J Radiol 2010;76:398-406.

7. Torabi M, Aquino SL, Harisinghani MG. Current concepts in lymph node imaging. J Nucl Med 2004;45:1509-18.

8. Plathow C, Walz M, Lichy MP, Aschoff P, Pfannenberg C, Bock H, Eschmann SM, Claussen CD, Schlemmer HP. Cost considerations for whole-body MRI and PET/CT as part of oncologic staging. Radiologe 2008;48:384-96.

9. Rabkin Z, Israel O, Keidar Z. Do hyperglycemia and diabetes affect the incidence of false-negative 18F-FDG PET/CT studies in patients evaluated for infection or inflammation and cancer? A Comparative analysis. J Nucl Med 2010;51:1015-20.

10. Takahara T, Imai Y, Yamashita T, Yasuda S, Nasu S, Van Cauteren M. Diffusion weighted whole body imaging with background body signal suppression (DWIBS): technical improvement using free breathing, STIR and high resolution 3D display. Radiat Med 2004;22:275-82.

11. Kharuzhyk S, Zhavrid E, Dziuban A, Sukolinskaja E, Kalenik O. Comparison of whole-body MRI with diffusion-weighted imaging and PET/CT in lymphoma staging. Eur Radiol 2020;30:3915-23.

12. Le Bihan D. Molecular diffusion nuclear magnetic resonance imaging. Magn Reson Q 1991;7:1-30.

13. Koh DM, Collins DJ. Diffusion-weighted MRI in the body: applications and challenges in oncology. AJR Am J Roentgenol 2007;188:1622-35.

14. Donners R, Blackledge M, Tunariu N, Messiou C, Merkle EM, Koh DM. Quantitative Whole-Body DiffusionWeighted MR Imaging. Magn Reson Imaging Clin N Am 2018;26:479-94.

15. Tunariu N, Blackledge M, Messiou C, Petralia G, Padhani A, Curcean S, Curcean A, Koh DM. What's New for Clinical Whole-body MRI (WB-MRI) in the 21st Century. Br J Radiol 2020;93:20200562.

16. Mayerhoefer ME, Karanikas G, Kletter K, Prosch H, Kiesewetter B, Skrabs C, Porpaczy E, Weber M, PinkerDomenig K, Berzaczy D, Hoffmann M, Sillaber C, Jaeger U, Müllauer L, Simonitsch-Klupp I, Dolak W, Gaiger A, Ubl P, Lukas J, Raderer M. Evaluation of diffusionweighted MRI for pretherapeutic assessment and staging of lymphoma: results of a prospective study in 140 patients. Clin Cancer Res 2014;20:2984-93.

17. Mayerhoefer ME, Karanikas G, Kletter K, Prosch H, Kiesewetter B, Skrabs C, Porpaczy E, Weber M, Knogler T, Sillaber C, Jaeger U, Simonitsch-Klupp I, Ubl P, Müllauer 
L, Dolak W, Lukas J, Raderer M. Evaluation of DiffusionWeighted Magnetic Resonance Imaging for Follow-up and Treatment Response Assessment of Lymphoma: Results of an 18F-FDG-PET/CT-Controlled Prospective Study in 64 Patients. Clin Cancer Res 2015;21:2506-13.

18. Fitzpatrick JJ, Ryan MA, Bruzzi JF. Diagnostic accuracy of diffusion-weighted imaging- magnetic resonance imaging compared to positron emission tomography/computed tomography in evaluating and assessing pathological response to treatment in adult patients with lymphoma: A systematic review. J Med Imaging Radiat Oncol 2018. [Epub ahead of print]. doi: 10.1111/1754-9485.12723.

19. Kwee TC, Ludwig I, Uiterwaal CS, van Ufford HM, Vermoolen MA, Fijnheer R, Bierings MB, Nievelstein RA. ADC measurements in the evaluation of lymph nodes in patients with non-Hodgkin lymphoma: feasibility study. MAGMA 2011;24:1-8.

20. Wang YJ, Xu XQ, Hu H, Su GY, Shen J, Shi HB, Wu FY. Histogram analysis of apparent diffusion coefficient maps for the differentiation between lymphoma and metastatic lymph nodes of squamous cell carcinoma in head and neck region. Acta Radiol 2018;59:672-80.

21. De Paepe KN, De Keyzer F, Wolter P, Bechter O, Dierickx D, Janssens A, Verhoef G, Oyen R, Vandecaveye $\mathrm{V}$. Improving lymph node characterization in staging malignant lymphoma using first-order ADC texture analysis from whole-body diffusion-weighted MRI. J Magn Reson Imaging 2018;48:897-906.

22. Blackledge MD, Collins DJ, Koh DM, Leach MO. Rapid development of image analysis research tools: Bridging the gap between researcher and clinician with pyOsiriX. Comput Biol Med 2016;69:203-12.

23. Blackledge MD, Koh DM, Collins DJ, Chua S, Leach MO. The utility of whole-body diffusion-weighted MRI for delineating regions of interest in PET. Nuclear instruments \& methods in physics research section a-accelerators spectrometers detectors and associated equipment 2013. p. 148-51.

24. Vezhnevets VK, V. "Grow-Cut" - Interactive MultiLabel N-D Image Segmentation". Proc. Graphicon; 2005. p. 150-6.

25. King AD, Ahuja AT, Yeung DK, Fong DK, Lee YY, Lei KI, Tse GM. Malignant cervical lymphadenopathy: diagnostic accuracy of diffusion-weighted MR imaging. Radiology 2007;245:806-13.

26. Abdel Razek AA, Soliman NY, Elkhamary S, Alsharaway MK, Tawfik A. Role of diffusion-weighted MR imaging in cervical lymphadenopathy. Eur Radiol 2006;16:1468-77.
27. Holzapfel K, Duetsch S, Fauser C, Eiber M, Rummeny EJ, Gaa J. Value of diffusion-weighted MR imaging in the differentiation between benign and malignant cervical lymph nodes. Eur J Radiol 2009;72:381-7.

28. Perrone A, Guerrisi P, Izzo L, D'Angeli I, Sassi S, Mele LL, Marini M, Mazza D. Diffusion-weighted MRI in cervical lymph nodes: differentiation between benign and malignant lesions. Eur J Radiol 2011;77:281-6.

29. Chen Y, Zhong J, Wu H, Chen N. The clinical application of whole-body diffusion-weighted imaging in the early assessment of chemotherapeutic effects in lymphoma: the initial experience. Magn Reson Imaging 2012;30:165-70.

30. Lin C, Itti E, Luciani A, Zegai B, Lin SJ, Kuhnowski F, Pigneur F, Gaillard I, Paone G, Meignan M, Haioun C, Rahmouni A. Whole-body diffusion-weighted imaging with apparent diffusion coefficient mapping for treatment response assessment in patients with diffuse large B-cell lymphoma: pilot study. Invest Radiol 2011;46:341-9.

31. Wu X, Kellokumpu-Lehtinen PL, Pertovaara H, Korkola P, Soimakallio S, Eskola H, Dastidar P. Diffusion-weighted MRI in early chemotherapy response evaluation of patients with diffuse large B-cell lymphoma--a pilot study: comparison with 2-deoxy-2-fluoro- D-glucose-positron emission tomography/computed tomography. NMR Biomed 2011;24:1181-90.

32. Wu X, Nerisho S, Dastidar P, Ryymin P, Järvenpää R, Pertovaara H, Eskola H, Kellokumpu-Lehtinen PL. Comparison of different MRI sequences in lesion detection and early response evaluation of diffuse large B-cell lymphoma--a whole-body MRI and diffusion-weighted imaging study. NMR Biomed 2013;26:1186-94.

33. Li S, Xue HD, Li J, Sun F, Jiang B, Liu D, Sun HY, Jin ZY. Application of whole body diffusion weighted MR imaging for diagnosis and staging of malignant lymphoma. Chin Med Sci J 2008;23:138-44.

34. De Paepe K, Bevernage C, De Keyzer F, Wolter P, Gheysens O, Janssens A, Oyen R, Verhoef G, Vandecaveye $\mathrm{V}$. Whole-body diffusion-weighted magnetic resonance imaging at 3 Tesla for early assessment of treatment response in non-Hodgkin lymphoma: a pilot study. Cancer Imaging 2013;13:53-62.

35. Herrmann K, Queiroz M, Huellner MW, de Galiza Barbosa F, Buck A, Schaefer N, Stolzman P, Veit-Haibach P. Diagnostic performance of FDG-PET/MRI and WBDW-MRI in the evaluation of lymphoma: a prospective comparison to standard FDG-PET/CT. BMC Cancer 2015;15:1002.

36. Maggialetti N, Ferrari C, Minoia C, Asabella AN, Ficco M, 
Loseto G, De Tullio G, de Fazio V, Calabrese A, Guarini A, Rubini G, Brunese L. Role of WB-MR/DWIBS compared to (18)F-FDG PET/CT in the therapy response assessment of lymphoma. Radiol Med 2016;121:132-43.

37. Siegel MJ, Jokerst CE, Rajderkar D, Hildebolt CF, Goyal S, Dehdashti F, Wagner Johnston N, Siegel BA. Diffusionweighted MRI for staging and evaluation of response in diffuse large B-cell lymphoma: a pilot study. NMR Biomed 2014;27:681-91.

38. Schob S, Münch B, Dieckow J, Quäschling U, Hoffmann KT, Richter C, Garnov N, Frydrychowicz C, Krause M, Meyer HJ, Surov A. Whole Tumor Histogram-profiling of Diffusion-Weighted Magnetic Resonance Images Reflects Tumorbiological Features of Primary Central Nervous System Lymphoma. Transl Oncol 2018;11:504-10.

39. Blackledge MD, Tunariu N, Orton MR, Padhani AR, Collins DJ, Leach MO, Koh DM. Inter- and Intra-

Cite this article as: Donners R, Yiin RSZ, Koh DM, De Paepe K, Chau I, Chua S, Blackledge MD. Wholebody diffusion-weighted MRI in lymphoma-comparison of global apparent diffusion coefficient histogram parameters for differentiation of diseased nodes of lymphoma patients from normal lymph nodes of healthy individuals. Quant Imaging Med Surg 2021;11(8):3549-3561. doi:10.21037/qims-21-50
Observer Repeatability of Quantitative Whole-Body, Diffusion-Weighted Imaging (WBDWI) in Metastatic Bone Disease. PLoS One 2016;11:e153840.

40. Sadinski M, Medved M, Karademir I, Wang S, Peng Y, Jiang Y, Sammet S, Karczmar G, Oto A. Short-term reproducibility of apparent diffusion coefficient estimated from diffusion-weighted MRI of the prostate. Abdom Imaging 2015;40:2523-8.

41. Moreau B, Iannessi A, Hoog C, Beaumont H. How reliable are ADC measurements? A phantom and clinical study of cervical lymph nodes. Eur Radiol 2018;28:3362-71.

42. Wu X, Pertovaara H, Dastidar P, Vornanen M, Paavolainen L, Marjomäki V, Järvenpää R, Eskola H, KellokumpuLehtinen PL. ADC measurements in diffuse large B-cell lymphoma and follicular lymphoma: a DWI and cellularity study. Eur J Radiol 2013;82:e158-64. 